\title{
AXL Downstream Targeting Unravels Synergistic Drug Combinations in Ovarian Carcinoma Cells
}

\author{
DESIRÈ M. ALAMPI ${ }^{1}$, EMILIO CIUSANI ${ }^{2}$, NIVES CARENINI ${ }^{1}$, \\ ELISABETTA CORNA ${ }^{1}$, LAURA GATTI ${ }^{3}$ and CRISTINA CORNO ${ }^{1}$ \\ ${ }^{1}$ Molecular Pharmacology Unit, Department of Applied Research and Technological Development, \\ Fondazione IRCCS Istituto Nazionale dei Tumori, Milan, Italy; \\ ${ }^{2}$ Neurological Biochemistry and Neuropharmacology, Fondazione IRCCS Istituto Neurologico Carlo Besta, Milan, Italy; \\ ${ }^{3}$ Cellular Neurobiology Laboratory, Cerebrovascular Unit, \\ Fondazione IRCCS Istituto Neurologico Carlo Besta, Milan, Italy
}

\begin{abstract}
Background: Platinum-based therapy represents the main pharmacological treatment for ovarian carcinoma. Since molecular targeting of receptor tyrosine kinases (RTK) affects factors that may modulate drug response, the aim of this study was to examine whether downstream targets of AXL RTK could be exploited to improve cell response to cisplatin. Materials and Methods: Inhibitors of p38 (SB203580) and of signal transducer and activator of transcription 3 (stattic) were employed in combination with cisplatin in ovarian carcinoma cell lines. Apoptosis assay and western blot analysis were performed to evaluate cell response after treatment. Results: SB203580 produced a synergistic effect in combination with cisplatin in cisplatin-resistant IGROV-1/PtI cells. In addition, a favorable drug interaction was observed in A2780 cells when pre-incubated with cisplatin prior to stattic. The analysis of cell response after combined treatment showed down-regulation of the pro-apoptotic protein BCL2-associated agonist of cell death (BAD). Conclusion: Our results support the notion that downstream targets of AXL in ovarian carcinoma cells can be exploited to increase cisplatin activity in ovarian carcinoma models.
\end{abstract}

Ovarian cancer is a frequent cause of cancer related deaths in women $(1,2)$, usually diagnosed as advanced disease, a feature associated with poor prognosis. The standard of care is surgery followed by systemic platinum-based chemotherapy (3). Despite a good clinical response to therapy, drug resistance often develops and few targeted agents have been introduced

Correspondence to: Cristina Corno, Molecular Pharmacology Unit, Department of Applied Research and Technological Development, Fondazione IRCCS Istituto Nazionale dei Tumori, Milan, Italy. Tel: +390223903080, e-mail: cristina.corno@istitutotumori.mi.it

Key Words: Ovarian carcinoma, cisplatin, AXL, p38 MAPK. into ovarian cancer treatment $(4,5)$. The knowledge gained on the molecular features of this disease has not been fully translated to the therapeutic setting. Therefore, further efforts are needed to define rational pharmacological approaches to combat aggressive ovarian carcinoma. Deregulation of survival pathways is a hallmark of cancer cells and contributes to tumor growth and progression (6). Receptor tyrosine kinases (RTK) are major players in tumor survival because they transduce signals related to cell growth, adhesion, differentiation, motility and survival $(7,8)$. AXL RTK, belonging to the TYRO 3AXL-MER (TAM) RTK subfamily, is overexpressed in hematological and solid malignancies $(9,10)$. Such a receptor has been proposed as a relevant therapeutic target for metastatic and advanced-stage human ovarian cancer (11). We reported that AXL is overexpressed in cisplatin-resistant ovarian carcinoma cells and is involved in sustaining increased invasive ability through activation of mitogen-activated protein kinase (MAPK) p38 and signal transducer and activator of transcription 3 (STAT3), as supported by the reduced activation of such factors following molecular targeting of AXL in several ovarian carcinoma cell lines (12).

Although AXL targeting may result in improved efficacy of selected antitumor agents (13), the use of small-molecule inhibitors of this kinase is challenging due to their promiscuity. Thus, selective inhibition of downstream targets of AXL in specific molecular backgrounds may lead to improved response to chemotherapy. In the present study, we investigated the possibility of improving the efficacy of cisplatin by inhibiting p38 or STAT3 in ovarian carcinoma cell lines. We observed schedule- and cell linedependent favorable interactions between cisplatin and the studied inhibitors. Our results support the notion of a protective role for $\mathrm{p} 38$ in drug-resistant ovarian carcinoma and suggest a regulatory function for STAT3 in cisplatinsensitive cells, strengthening the need for active drug development in this field. 


\section{Materials and Methods}

Drugs. Cisplatin (Teva Pharma Italia S.r.l., Milan, Italy) was diluted in saline. Stattic (Selleck Chemicals, Aurogene Srl, Rome, Italy) and SB203580 (Sigma-Aldrich, St. Louis, MO, USA) were dissolved in dimethyl sulfoxide and diluted in culture medium (final dimethyl sulfoxide concentration $0.25 \%$ ).

Cell lines and cell sensitivity to drugs. The human ovarian carcinoma cell lines IGROV-1 and A2780, and the cisplatin-resistant variant IGROV-1/Pt1 obtained as previously described (12), were cultured in RPMI-1640 medium (Lonza, Basel, Switzerland), supplemented with $10 \%$ fetal bovine serum (Gibco, Life Technologies, Carlsbad, CA, USA). Cells were thawed from frozen stocks and cultured for no more than 20 passages. Cell sensitivity to drugs was measured by growth-inhibition assays, evaluated in keeping with cell pharmacology guidelines (14). Twenty-four hours after seeding, cells were exposed to the drugs for $72 \mathrm{~h}$, according different schedules of treatments (simultaneously, $24 \mathrm{~h}$ preincubation with cisplatin or 24-h pre-incubation with p38/STAT3 inhibitors). Cells were counted with a cell counter at the end of drug incubation. $\mathrm{IC}_{50}$, defined as the drug concentration producing $50 \%$ decrease of cell growth, was determined. The type of drug interaction was calculated according to the Chou-Talalay method using Calcusyn software (Biosoft, Cambridge, UK), which assigns a combination index value (CI) to a drug combination (15). A CI value lower than $0.85-0.90$ indicates synergistic drug interactions; CI values of 1.20-1.45 and around 1 represent antagonistic and additive effects, respectively. For each assay, at least three independent experiments were performed.

Western blot analysis. Western blot analysis of proteins involved in cell response to drug combination treatment was carried out as described elsewhere (16). Immunoreactive bands were revealed by enhanced chemiluminescence detection system ECL (GE Healthcare, Little Chalfont, UK). The primary antibodies used in the present study included: anti-AXL, anti-p38 and anti-phosphop38 (Thr180/Tyr182) from Cell Signaling (Danvers, MA, USA), anti-BCL2-associated agonist of cell death (BAD) and antiphospho-BAD (Ser 112) from Cell Signaling; anti-BAX, antitubulin and anti-vinculin from Sigma-Aldrich; anti-p53 from BD Biosciences (Milan, Italy), anti-STAT3 from SignalChem (Richmond, Canada) and anti-phospho STAT3 (Tyr705) from Thermo Fisher Scientific (Monza, Italy). Secondary antibodies were purchased from GE Healthcare (Little Chalfont, Buckinghamshire UK). Band intensities were quantified by scanning films and processing image intensities with Image J $1.47 \mathrm{v}$ software (Bethesda, MD, USA).

Analysis of apoptosis. Apoptosis was evaluated by annexin Vbinding assay (Immunostep, Salamanca, Spain), as previously described (17), according to the schedule used for cell-sensitivity assay.

Statistical analysis. Statistical analyses were performed using GraphPad Prism $^{\mathrm{TM}}$ software (GraphPad Software, San Diego, CA, USA). For comparison of $\mathrm{IC}_{50}$ values, ANOVA followed by Bonferroni's multiple comparison test was used. For annexin V binding assays, ANOVA followed by Tukey's multiple comparison test was used to compare different samples.

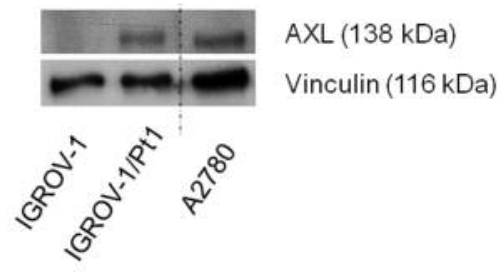

Figure 1. Western blot analysis of AXL receptor tyrosine kinase expression in ovarian carcinoma cell lines. The dashed line refers to the removal of irrelevant lanes from the image of the blot. Control loading is shown by vinculin. The AXL band intensity was quantified using ImageJ, normalized to that of the loading control and expressed relative to the level of control cells (set to 1). Relative expression AXL was 60.17 and 64.43 for IGROV-1/PtI and A2780 cells, respectively.

Table I. Sensitivity of ovarian carcinoma cells to cisplatin and targeting compounds. Cells were seeded and 24 h later exposed to cisplatin, p38 inhibitor SB203580, and inhibitor of signal transducer and activator of transcription 3 stattic. $I C_{50}$ is the concentration inhibiting cell growth by $50 \%$.

\begin{tabular}{lrrr}
\hline & \multicolumn{3}{c}{$\mathrm{IC}_{50}(\mu \mathrm{M})$} \\
\cline { 2 - 4 } Compound & IGROV-1 & IGROV-1/Pt1 & A2780 \\
\hline Cisplatin & $0.55 \pm 0.06$ & $7.80 \pm 3.1$ & $0.082 \pm 0.02$ \\
SB203580 & $19.62 \pm 1.50$ & $21.75 \pm 6.6$ & $21.10 \pm 2.90$ \\
Stattic & $4.26 \pm 1.50$ & $5.37 \pm 0.5$ & $0.420 \pm 0.08$ \\
\hline
\end{tabular}

\section{Results}

Features of ovarian carcinoma cell lines. We used ovarian carcinoma cell lines previously profiled for the expression of AXL RTK at the mRNA and protein levels (12), namely IGROV-1 cells, the IGROV-1/Pt1 cisplatin-resistant variant and the A2780 cell line (Figure 1). Cell sensitivity to cisplatin and to compounds inhibiting p38 (SB203580) and STAT3 (stattic) was examined by growth-inhibition assays after 72-h exposure (Table I). The IGROV-1/Pt1 variant is characterized by cisplatin resistance, with a degree of resistance of 14-fold, whereas A2780 cells are more sensitive to cisplatin than IGROV-1 cells as shown by their $\mathrm{IC}_{50}$ values (ANOVA, $p<0.05$ Bonferroni's multiple comparison test). The p38 inhibitor displayed similar ability to inhibit growth in the three cell lines, whereas stattic was more potent against A2780 cells than the other cell lines (ANOVA, $p<0.05$ Bonferroni's multiple comparison test).

Drug combination studies. Because molecular targeting of AXL by small interfering RNA (siRNA) transfection resulted in reduced activation of p38 in ovarian carcinoma cells expressing AXL (12), cellular sensitivity to cisplatin in combination with the p38 inhibitor SB203580 was tested by growth-inhibition assays using a simultaneous 72-h treatment 
Table II. Effect of the combination of cisplatin and p38 inhibitor SB203580 on IGROV-1/Pt1 cells. Cells were co-incubated with cisplatin and SB203548 for $72 \mathrm{~h}$. The combination index values (CI) obtained with 3 and $10 \mu M$ SB203580 combined with different cisplatin concentrations are shown. The reported values are the mean $\pm S D$ of three independent experiments.

\begin{tabular}{lcc}
\hline SB203580 $(\mu \mathrm{M})$ & Cisplatin $(\mu \mathrm{M})$ & $\mathrm{CI}( \pm \mathrm{SD})$ \\
\hline 3 & 0.3 & $0.184 \pm 0.04$ \\
3 & 1.0 & $0.344 \pm 0.10$ \\
3 & 3.0 & $0.414 \pm 0.10$ \\
3 & 10 & $0.602 \pm 0.10$ \\
10 & 0.3 & $0.305 \pm 0.10$ \\
10 & 1.0 & $0.393 \pm 0.30$ \\
10 & 3.0 & $0.658 \pm 0.40$ \\
10 & 10 & $0.770 \pm 0.30$ \\
\hline
\end{tabular}

Interaction: $\quad \mathrm{CI}<0.85-0.90$ synergistic; $1.20-1.45$ antagonistic; $\sim 1$ additive effects.

Table III. Effect of the combination of cisplatin and stattic on A2780 cells. Cells were $24 \mathrm{~h}$ pre-incubated with cisplatin and then coincubated with stattic (inhibitor of signal transducer and activator of transcription 3) for $48 \mathrm{~h}$. The combination index (CI) values obtained with $0.3 \mu M$ stattic combined with different cisplatin concentrations are shown. The reported values are the mean $\pm S D$ of three independent experiments.

\begin{tabular}{lcr}
\hline Stattic $(\mu \mathrm{M})$ & Cisplatin $(\mu \mathrm{M})$ & CI $( \pm \mathrm{SD})$ \\
\hline 0.3 & 0.03 & $1.0 \pm 0.60$ \\
0.3 & 0.10 & $0.75 \pm 0.01$ \\
0.3 & 0.30 & $0.63 \pm 0.10$ \\
0.3 & 1.00 & $1.0 \pm 0.50$ \\
\hline
\end{tabular}

Interaction: CI $<0.85-0.90$ synergistic; $1.20-1.45$ antagonistic; $~ 1$ additive effects.

schedule in cells expressing marked levels of AXL, namely the IGROV-1/Pt1 and A2780 cell lines. In IGROV-1/Pt1 cells, cisplatin was combined with 3 and $10 \mu \mathrm{M} \mathrm{SB} 203580$, which per se produced an inhibition of cell growth by $45 \%$ and $30 \%$, respectively. In this cisplatin-resistant subline, we observed a synergistic interaction between cisplatin and the p38 inhibitor as evidenced by the CI values, which were below 0.85 under all conditions (Table II; Figure 2).

No synergistic interaction was obtained against the parental cell line, which expresses low levels of AXL, nor for the A2780 cell line, with CI values consistently greater than 1 (data not shown).

Molecular targeting of AXL has been shown to reduce activation of STAT3 (12). Therefore, cellular sensitivity to cisplatin was tested in combination with an inhibitor of STAT3, stattic. In IGROV-1 and IGROV-1/Pt1 cells, no favorable interaction was observed with different schedule
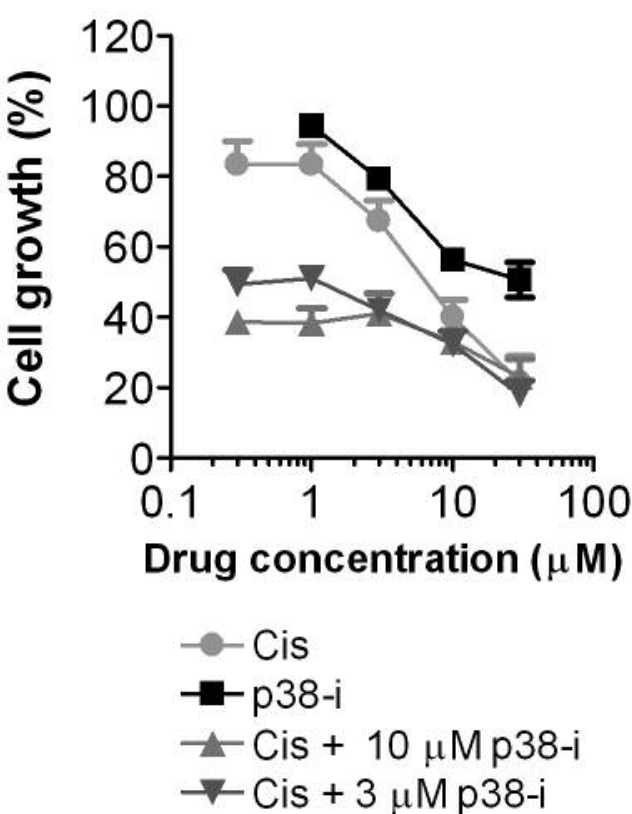

Figure 2. Sensitivity of IGROV-1/Pt1 cells to the combination of cisplatin (Cis) and the p38 inhibitor (p38-i) SB203580. Cells were seeded and 24 h later exposed to cisplatin alone, SB203580 alone or to the simultaneous combination of cisplatin and the p38 inhibitor (3 or $10 \mu M)$ for $72 \mathrm{~h}$. The reported values are the mean $\pm S D$ of three independent experiments.

of treatment (data not shown), the CI values being mostly greater than 1. A more favorable drug interaction with 24-h pre-incubation with cisplatin followed by 48-h co-incubation with stattic was observed for A2780 cells exposed to differents concentration of cisplatin and $0.3 \mu \mathrm{M}$ stattic, the CI value being around 0.6 and 0.7 for two combinations (Table III).

Ovarian carcinoma cell response to drug combination treatment. Response to the drug combinations was examined in IGROV-1/Pt1 and A2780 cells, selected based on the expression of AXL, with reference to apoptosis occurrence and to modulation of proteins involved in apoptosis. In IGROV-1/Pt1 cells, we found that the combination of cisplatin and p38 inhibitor SB203580 induced slightly higher levels of apoptosis than cisplatin alone, when cells were harvested $48 \mathrm{~h}$ after drug exposure and apoptosis levels were quantified by annexin $\mathrm{V}$ binding assays (Figure 3A). Western blot analysis indicated that cisplatin alone and in combination with $\mathrm{p} 38$ inhibitor resulted in down-regulation of the BCL2 family protein BAD (Figure 3B), whose phosphorylation was low and not assessable (data not shown). Increased levels of the pro-apoptotic protein BAX and of p53 were also observed in all treated cells. In A2780 cells exposed $24 \mathrm{~h}$ to cisplatin and then co-incubated with 

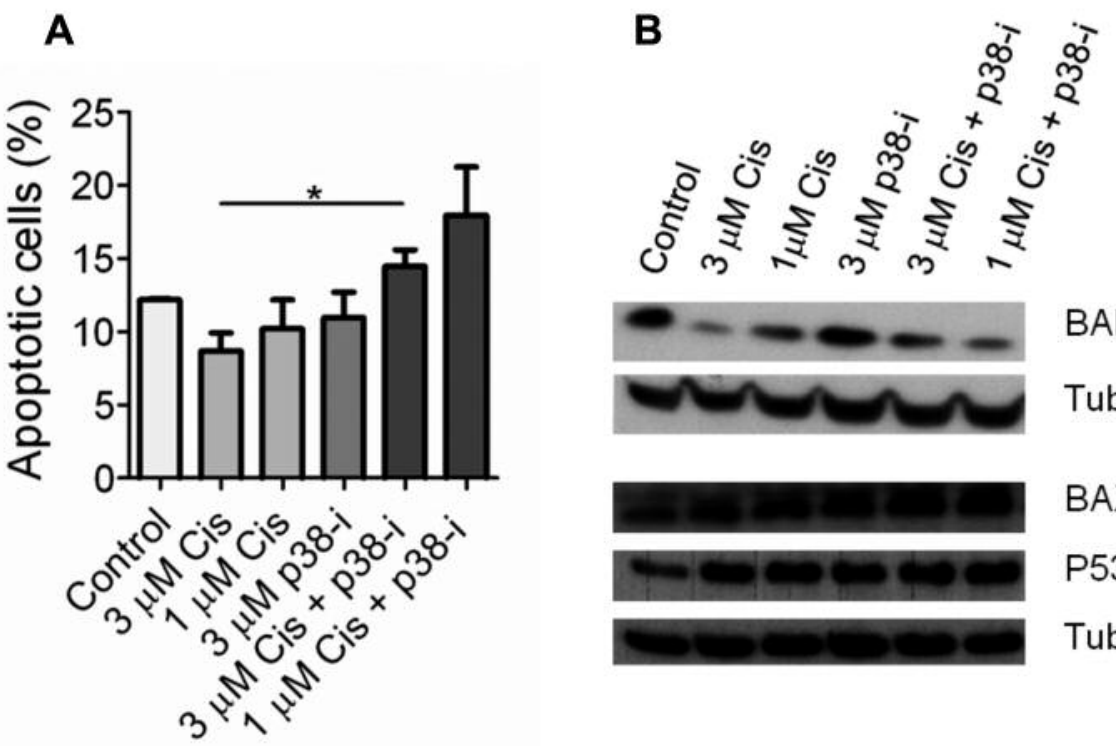

$\operatorname{BAD}(23 \mathrm{kDa})$

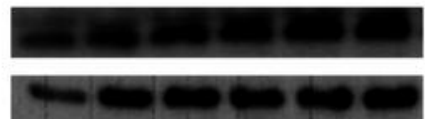

Tubulin (56 kDa)

$\operatorname{BAX}(20 \mathrm{kDa})$

P53 (53 kDa)

Tubulin (56 kDa)

Figure 3. Response of IGROV-1/Pt1 cells to the combination of cisplatin (Cis) and the p38 inhibitor SB203580. A: Drug-induced apoptosis of IGROV-1/Pt1 cells exposed for $48 \mathrm{~h}$ to the combination of cisplatin and the p38 inhibitor (p38-i) SB203580. The columns indicate late apoptotic (annexin V-propidium iodide-positive) cells. The values represent the mean $\pm S D, n=3$. ${ }^{*}$ Significantly different at $p<0.05$ by one-way ANOVA. B: Western blot analysis of apoptosis-related proteins in IGROV-1/Pt1 cells, treated for $48 \mathrm{~h}$ with cisplatin and SB203580. Control loading is shown by tubulin. Band intensity was quantified using ImageJ, normalized to that of the loading control and expressed relative to the level of control cells (set to 1). Normalized values corresponding to $3 \mu \mathrm{M}$ cisplatin, $1 \mu \mathrm{M}$ cisplatin, $3 \mu \mathrm{M}$ p38-I, $3 \mu \mathrm{M}$ cisplatin plus p38-I, $1 \mu \mathrm{M}$ cisplatin plus p38-I were $0.30,0.58,0.83,0.52,0.25$ for $B A D ; 1.62,1.83,2.16,1.77,1.55$ for $B A X$; and 2.41, 2.12, 1.88, 1.81, 2.1 for p53.
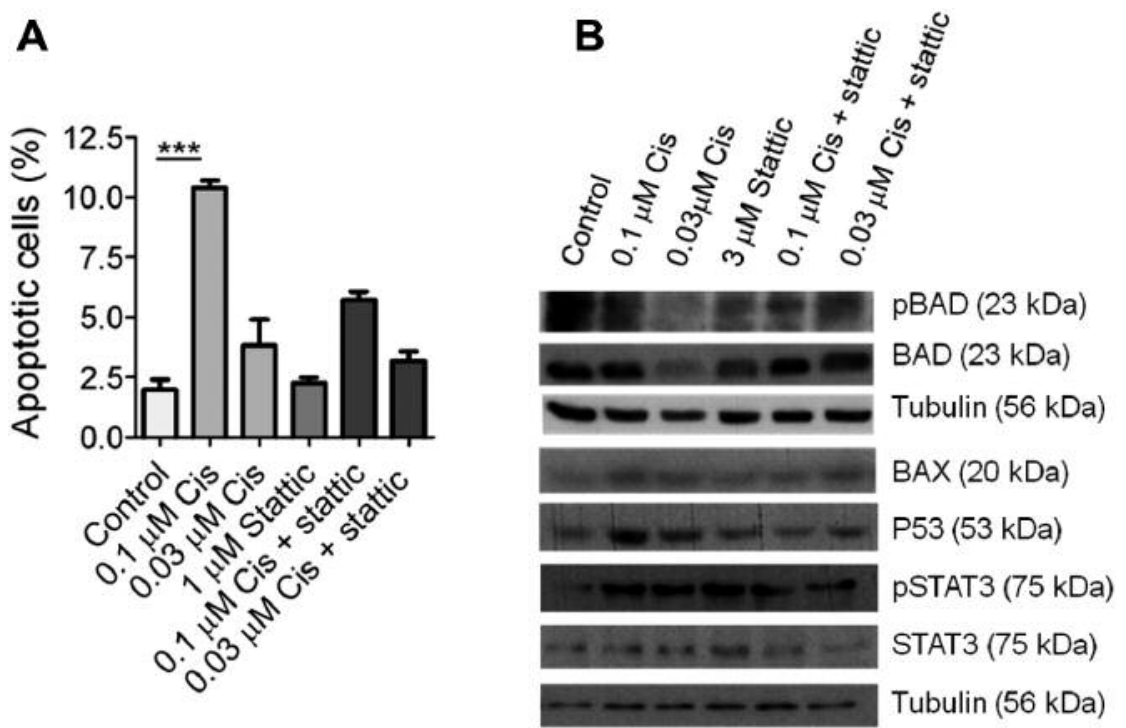

Figure 4. Response of A2780 cells to the combination of cisplatin (Cis) and the signal transducer and activator of transcription 3 (STAT3) inhibitor stattic. A: Drug-induced apoptosis of A2780 cells exposed for $24 \mathrm{~h}$ to cisplatin followed by $24 \mathrm{~h}$ co-incubation with stattic. The columns indicate late apoptotic (annexin V-propidium iodide-positive) cells. The values represent the mean $\pm S D, n=3$. ***Significantly different at $p<0.001$ by oneway ANOVA. B: Western blot analysis of apoptosis-related proteins in A2780 cells, treated for $48 \mathrm{~h}$ with cisplatin and stattic, as in (A). Control loading is shown by tubulin. Band intensity was quantified using ImageJ, normalized to that of the loading control and expressed relative to the level of control cells (set to 1). Normalized expression corresponding to $0.1 \mu \mathrm{M}$ cisplatin, $0.03 \mu \mathrm{M}$ cisplatin, $3 \mu \mathrm{M}$ stattic, $0.1 \mu \mathrm{M}$ cisplatin plus stattic, $0.03 \mu \mathrm{M}$ cisplatin plus stattic were 1.66, 0.56, 1.06, 1.35, 1.79 for $p-B A D ; 1.24,0.71,1.24,1.53,1.4$ for BAD; 6.5, 0.9, 0.62, $1.12,1.83$ for $B A X ; 4.4,0.9,0.7,0.34,0.46$ for $p 53 ; 3.71,4.01,3.30,1.97,2.38$ for $p$-STAT3; and 0.89, 1.03, 1.06, 0.67, 0.34 for STAT3. 
the STAT3 inhibitor stattic. Cisplatin alone was capable of triggering marked levels of apoptosis, as evidenced by annexin $\mathrm{V}$ binding assay $(p<0.001$, by one-way ANOVA; Figure 4A). Cisplatin reduced BAD phosphorylation as shown by western blot analysis and lower levels of BAD were observed in cisplatin-treated cells. BAX and p53 were also slightly up-modulated by cisplatin exposure. All treatments increased phospho-STAT3 levels but the effect was less pronounced upon combined treatments (Figure 4B).

\section{Discussion}

The occurrence of drug resistance, often associated with deregulation of cell survival pathways and increased metastatic cell ability, is a major clinical issue limiting the efficacy of antitumor agents (18). In the present study, we employed ovarian carcinoma cell lines expressing AXL RTK (namely IGROV-1/Pt1 and A2780) to determine whether targeting AXL downstream signaling may be a useful strategy for improving sensitivity to cisplatin, which represents the mainstay of first-line treatment for ovarian carcinoma. In a previous study, we found that p38 and STAT3 are downstream targets of AXL, as shown by a lossof-function approach (12). When assaying the effect of cisplatin in combination with the p38 inhibitor SB203580 in IGROV-1/Pt1 cells, we found a marked synergistic interaction, not evident in the parental IGROV-1 cells nor in the A2780 cell line. The observed synergy was associated with increased apoptosis, as indicated by a higher percentage of apoptotic cells when treated with the cisplatin-p38 inhibitor combination as compared to single agent-treatment. This finding suggests the possibility of inducing apoptosis in p53-mutant cells (in this case IGROV-1/Pt1 cells) by interfering with p38 activation. Indeed, it appears that in these cells, p38 plays a protective role against cisplatininduced damage and its inhibition leads to cell death, consistent with the modulation of proteins implicated in apoptosis induction as evidenced by western blot analysis. Specifically, we observed reduced levels of the BCL2 family member BAD after IGROV-1/Pt1 cells were exposed to cisplatin alone, and or in combination with the p38 inhibitor SB203580.

Our results are in keeping with reports showing a protective role for p38 against DNA-damaging agents. For instance, in renal tubular epithelial cells inhibition of p38MAPK potentiates cisplatin-induced apoptosis through depletion of reduced glutathione and an increase in drug accumulation (19). Moreover, a favorable effect of p38 inhibition has been reported in cervical cancer models following inhibition of aurora kinases (20). A protective role for p38 against DNA damage is supported also by studies in colon cancer cells exposed to DNA topoisomerase I inhibitors (21).
In the present study, we also observed a favorable drug combination between cisplatin and the STAT3 inhibitor stattic in the A2780 cell line, but not in other cell lines. We identified a favorable drug interaction when these cells were exposed to cisplatin before co-incubation with stattic. Striking apoptosis induction was readily apparent upon A2780 cell exposure to cisplatin alone. In A2780 cells, the pro-apoptotic ability of cisplatin was superior to its combination with stattic, suggesting that the combination might only inhibit cell growth without killing the cells. Consistent with this, western blot analysis indicated that cisplatin was capable of down-regulating BAD and phosphorylaton of Ser112 of BAD, a condition expected to favor apoptosis. In fact, BAD phosphorylation, known to result from activation of the phosphatidylinositol-4,5bisphosphate 3-kinase/AKT serine/threonine kinase 1 signaling pathway, is needed to suppress apoptosis and maintain cell survival (22).

The design of rational drug combinations involving chemotherapeutic agents is still a promising research area (23), and our results support the interest of p38 inhibitors as sensitizers to use against cisplatin-resistant ovarian carcinoma.

\section{Conflicts of Interest}

The Authors declare no conflict of interest in regard to this study.

\section{Authors' Contributions}

Investigation: Cristina Corno, Desirè Alampi, Emilio Ciusani, Nives Carenini and Elisabetta Corna; Methodology, Nives Carenini and Elisabetta Corna. Supervision: Laura Gatti and Cristina Corno. Writing - original draft: Cristina Corno.

\section{Acknowledgements}

This study was supported by a grant to Paola Perego (Molecular Pharmacology Unit, Department of Applied Research and Technological Development, Fondazione IRCCS Istituto Nazionale dei Tumori, via Amadeo 42, 20133 Milan, Italy) from the Associazione Italiana per la Ricerca sul Cancro (AIRC-IG 15333).

\section{References}

1 Siegel RL, Miller KD and Jemal A: Cancer Statistics, 2018. CA Cancer J Clin 68: 7-30, 2018. PMID: 29313949. DOI: 10.3322/ caac. 21442

2 Eisenhauer EA: Real-world evidence in the treatment of ovarian cancer. Ann Oncol 28: viii61-viii65, 2017. PMID: 29232466. DOI: $10.1093 /$ annonc/mdx443

3 Herzog TJ and Monk BJ: Bringing new medicines to women with epithelial ovarian cancer: What is the unmet medical need? Gynecol Oncol Res Pract 4: 13-017-0050-0, 2017. PMID: 28904804. DOI: $10.1186 / \mathrm{s} 40661-017-0050-0$

4 Cortesi L, Toss A and Cucinotto I: PARP Inhibitors for the treatment of ovarian cancer. Curr Cancer Drug Targets 18(9): 877-893, 2018. PMID: 29521233. DOI: $10.2174 / 15680096186$ 66180308104646 
5 McLachlan J, George A and Banerjee S: The current status of PARP inhibitors in ovarian cancer. Tumori 102(5): 433-440, 2016. PMID: 27716873. DOI: $10.5301 / \mathrm{tj} .5000558$

6 Hanahan D and Weinberg RA: Hallmarks of cancer: The next generation. Cell 144(5): 646-674, 2011. PMID: 21376230. DOI: 10.1016/j.cell.2011.02.013

7 Cassinelli G, Zuco V, Gatti L, Lanzi C, Zaffaroni N, Colombo $\mathrm{D}$ and Perego P: Targeting the Akt kinase to modulate survival, invasiveness and drug resistance of cancer cells. Curr Med Chem 20(15): 1923-Ia45, 2013. PMID: 23410153.

8 Samatar AA and Poulikakos PI: Targeting RAS-ERK signalling in cancer: promises and challenges. Nat Rev Drug Discov 13(12): 928-942, 2014. PMID: 25435214. DOI: $10.1038 /$ nrd 4281

9 Corno C, Gatti L, Lanzi C, Zaffaroni N, Colombo D and Perego P: Role of the receptor tyrosine kinase Axl and its targeting in cancer cells. Curr Med Chem 23(15): 1496-1512, 2016. PMID: 27048336.

10 Gay CM, Balaji $\mathrm{K}$ and Byers LA: Giving AXL the axe: Targeting AXL in human malignancy. Br J Cancer 116(4): 415423, 2017. PMID: 28072762. DOI: 10.1038/bjc.2016.428

11 Rankin EB, Fuh KC, Taylor TE, Krieg AJ, Musser M, Yuan J, Wei K, Kuo CJ, Longacre TA and Giaccia AJ: AXL is an essential factor and therapeutic target for metastatic ovarian cancer. Cancer Res 70(19): 7570-7579, 2010. PMID: 20858715. DOI: $10.1158 / 0008-5472 . C A N-10-1267$

12 Corno C, Gatti L, Arrighetti N, Carenini N, Zaffaroni N, Lanzi $\mathrm{C}$ and Perego P: AXL molecular targeting counteracts aggressiveness but not platinum-resistance of ovarian carcinoma cells. Biochem Pharmacol 136: 40-50, 2017. PMID: 28404378. DOI: $10.1016 /$ j.bcp.2017.04.002

13 Schoumacher M and Burbridge M: Key Roles of AXL and MER receptor tyrosine kinases in resistance to multiple anticancer therapies. Curr Oncol Rep 19(3): 19, 2017. PMID: 28251492. DOI: $10.1007 / \mathrm{s} 11912-017-0579-4$

14 Perego P, Hempel G, Linder S, Bradshaw TD, Larsen AK, Peters GJ, Phillips RM and EORTC PAMM Group: Cellular pharmacology studies of anticancer agents: recommendations from the EORTCPAMM group. Cancer Chemother Pharmacol 81(3): 427-441, 2018. PMID: 29285635. DOI: $10.1007 / \mathrm{s} 00280-017-3502-7$

15 Chou TC: Drug combination studies and their synergy quantification using the Chou-Talalay method. Cancer Res 70(2): 440-446, 2010. PMID: 20068163. DOI: 10.1158/0008-5472. CAN-09-1947.

16 Cossa G, Lanzi C, Cassinelli G, Carenini N, Arrighetti N, Gatti L, Corna E, Zunino F, Zaffaroni N and Perego P: Differential outcome of MEK1/2 inhibitor-platinum combinations in platinum-sensitive and -resistant ovarian carcinoma cells. Cancer Lett 347(2): 212-224, 2014. PMID: 24576622. DOI: 10.1016/ j.canlet.2014.02.016.
17 Corno C, Stucchi S, De Cesare M, Carenini N, Stamatakos S, Ciusani E, Minoli L, Scanziani E, Argueta C, Landesman Y, Zaffaroni N, Gatti L and Perego P: FoxO-1 contributes to the efficacy of the combination of the XPO1 inhibitor selinexor and cisplatin in ovarian carcinoma preclinical models. Biochem Pharmacol 147: 93-103, 2018. PMID: 29155058. DOI: 10.1016/ j.bcp.2017.11.009

18 Alexander S and Friedl P: Cancer invasion and resistance: interconnected processes of disease progression and therapy failure. Trends Mol Med 18(1): 13-26, 2012. PMID: 22177734. DOI: 10.1016/j.molmed.2011.11.003

19 Rodríguez-García ME, Quiroga AG, Castro J, Ortiz A, Aller P and Mata F: Inhibition of p38-MAPK potentiates cisplatininduced apoptosis via GSH depletion and increases intracellular drug accumulation in growth-arrested kidney tubular epithelial cells. Toxicol Sci 111(2): 413-423, 2009. PMID: 19578154. DOI: 10.1093/toxsci/kfp145.

20 Jin X, Mo Q, Zhang Y, Gao Y, Wu Y, Li J, Hao X, Ma D, Gao $\mathrm{Q}$ and Chen P: The p38 MAPK inhibitor BIRB796 enhances the antitumor effects of VX680 in cervical cancer. Cancer Biol Ther 17(5): 566-576, 2016. PMID: 27082306. DOI: 10.1080/1538 4047.2016.1177676

21 Paillas S, Causse A, Marzi L, de Medina P, Poirot M, Denis V, Vezzio-Vie N, Espert L, Arzouk H, Coquelle A, Martineau P, Del Rio M, Pattingre S and Gongora C: MAPK14/p38 $\alpha$ confers irinotecan resistance to TP53-defective cells by inducing survival autophagy. Autophagy 8(7): 1098-1112, 2012. PMID: 22647487. DOI: 10.4161/auto.20268 PMID: 22647487

22 Datta SR, Dudek H, Tao X, Masters S, Fu H, Gotoh Y and Greenberg ME: Akt phosphorylation of BAD couples survival signals to the cell-intrinsic death machinery. Cell 91(2): 231-241, 1997. PMID: 9346240.

23 Masood I, Kiani MH, Ahmad M, Masood MI and Sadaquat H: Major contributions towards finding a cure for cancer through chemotherapy: A historical review. Tumori 102(1): 6-17, 2016. PMID: 26350183. DOI: 10.5301/tj.5000387 